\title{
Distortion of non-invasive cardiac pulse curves A capillary-damped pick-up and a calibration unit for apex cardiograms and other pulse curves
}

\author{
JOHN WIKSTRAND, KENTH NILSSON, AND INGEMAR WALLENTIN \\ From the Department of Clinical Physiology, Sahlgren's Hospital, University of Göteborg and the \\ Department of Electromedicine, Siemens-Elema AB, Solna, Sweden
}

A calibration unit and a capillary-damped pick-up, of funnel type, intended for apex cardiograms and other non-invasive cardiac pulse curves are presented. The physical properties of a recording system, suitable for non-invasive cardiac diagnostic techniques, are reviewed.

In 4 identical recording units consisting of a funnel with and without capillary damping, connected via air transmission to a transducer, the overamplification in the underdamped systems varied between 2.4 and $4 \cdot 3$, and in the capillary-damped systems between $1 \cdot 1$ and 1.2. The capillary damping gave an approximately linear relation between phase shift and frequency in the high frequency range with a calculated delay of about 4 ms.

The low frequency time constant in the systems varied between 1.9 and $4.6 \mathrm{~s}$, depending on the amplifier setting used, which for sine curves of frequency 0.5 and $2 \mathrm{~Hz}$ means an apparent prematurity for their maxima and minima varying between 53 and $1 \mathrm{~ms}$, this being larger with lower frequency time constants and lower frequency curves.

During recent years non-invasive evaluation of cardiac function has attracted great interest. By recording the carotid arterial pulse, apex cardiogram, and jugular venous pulse, often in combination with an electrocardiogram and phonocardiogram, a considerable number of variables has been studied. These include curve configuration, certain relative amplitudes, such as the $a$ wave percentage amplitude of the total deflection of the apex cardiogram, curve derivatives, and systolic and diastolic time intervals.

During respiration the variation of the pulse curves around the baseline is pronounced. The pulse curves are, therefore, recorded during relaxed expiratory apnoea, which means that the time available for continuous recording is limited. If the low-frequency time constant is over $4 \mathrm{~s}$, practical difficulties may arise, since the low frequency time constant governs the time taken for a pulse curve to centre around the baseline. It is a well-known fact that too short a low frequency time constant, resulting from the properties of the measuring system or temporary air leakage, distorts the low Received for publication 5 January 1977 frequency parts of the curves. In one and the same curve a short low frequency time constant may give erroneous results for one variable but not for another (Johnson et al., 1971). Thus the low frequency time constant must not be too short when low frequency parts of the curves are to be reproduced. On the other hand, for the high frequency parts of a curve, e.g. the systolic upstroke in the apex cardiogram, it is the reproduction of the upper frequency range that must be good. The requirements which therefore should be fulfilled have been little studied and apart from the low frequency time constant little attention has been paid to the properties of the recording system used for noninvasive pulse curves.

In our laboratory, interest in the possibility of calibrating our pulse curves and studying the derivatives of the apex cardiogram and carotid pulse tracing was awakened by the reports of Reale (1967), Sutton and Craige (1967), and Sutton et al. (1970). A calibration unit was constructed (Siemens-Elema AB, Sweden). It was then realised that the funnel type pick-up used for recording the pulse curves was underdamped, causing serious 
distortion of the first derivative of the calibration signal. A capillary-damped funnel pick-up was constructed (Kenth Nilsson, Siemens-Elema AB, Sweden). This funnel has now been used to record over 10000 curves and we have found it usable for all sorts of pulse curves. The pick-up is now generally available (Siemens-Elema AB, Sweden). The properties of this capillary-damped pick-up and transducer are described in this paper and compared with the system used earlier. The calibration unit is described and the physical principles of systems used for recording pulse curves in non-invasive cardiology are reviewed.

\section{Theoretical background}

The apex cardiogram can, in stylised form, be approximated by a square wave. A square wave has a rapid upstroke, a plateau, a rapid downstroke, a plateau, and so on. The system of recording should reproduce both the rapid changes and the plateaux correctly. Proper reproduction of the rapid changes by the system of recording demands good properties within the high frequency range, otherwise the curve will show overshoot or slow response. Proper reproduction of the plateaux, on the other hand, demands good properties within the low frequency range, otherwise the curve will show a fall towards the baseline (Fig. 1). This fall is called 'sag' and its magnitude is determined by the low frequency time constant, the sag being larger the shorter the low frequency time constant. Moreover, in every linear system (as ours are assumed to be), a sag in the reproduction of a plateau is always followed by an undershoot of the reproduced curve below the following baseline that is as large as the sag; it is in practice very often this inevitable undershoot, rather than the sag itself, that is the most troublesome aspect of poor reproduction of the plateau (see below, discussion of Fig. 1).

The analysis in this paper is divided into two parts, one describing the distortion within the high frequency range, for the rapidly changing parts of the curves, and the other describing the distortion within the low frequency range, for the slowly changing parts.

The description concerns the specific system used in our group, consisting of a funnel-shaped pick-up connected via air transmission to a crystal transducer. This system is, however, representative of most recording systems for pulse curves used in non-invasive cardiology.

The pulse curves are general time functions. To examine how rapid changes in these time functions

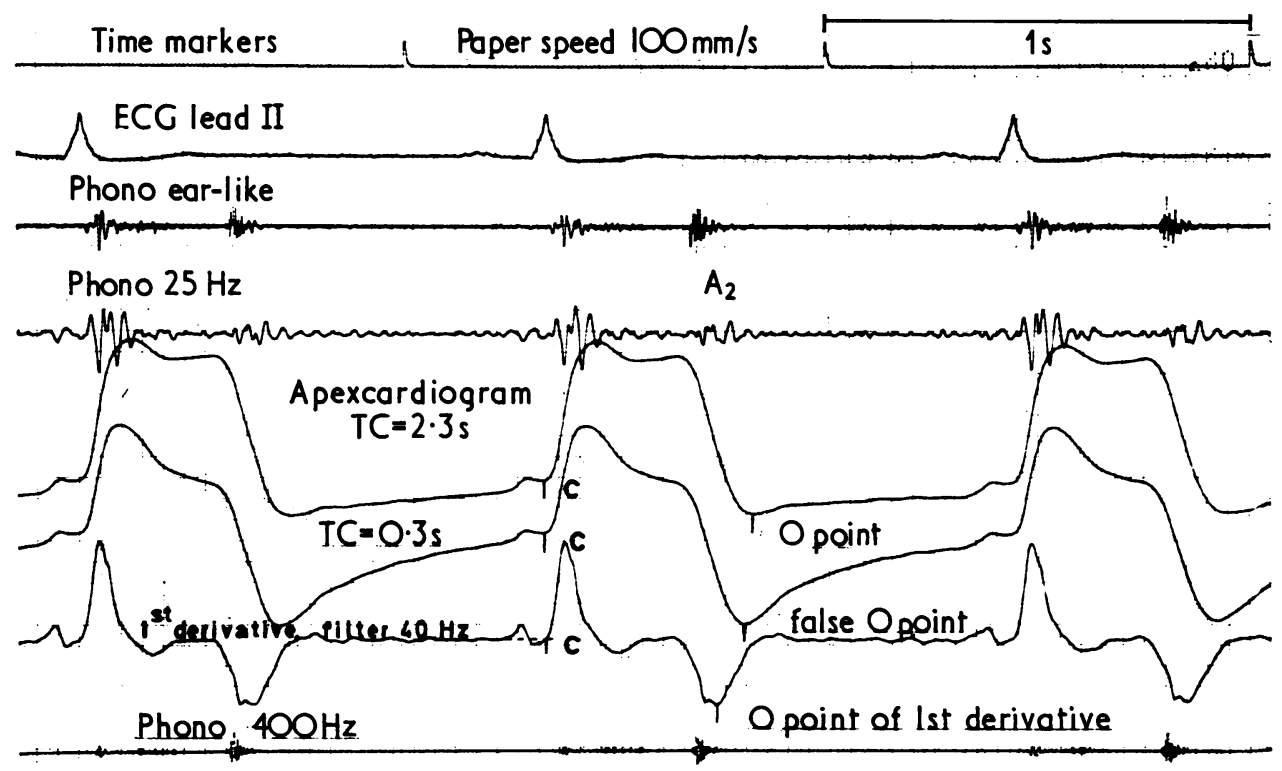

Fig. 1 Apex cardiogram recorded with low frequency time constant (TC $2.3 \mathrm{~s}$ ) and with curve processing giving a low frequency time constant of $0.3 \mathrm{~s}$ and the first derivative of the apex cardiogram. The electrocardiogram lead II and phonocardiogram (ear-like, 25 and $400 \mathrm{~Hz}$ ) are recorded simultaneously. Note that the $O$ point in the fifth channel $(T C=0.3 \mathrm{~s}$ ) is identified about $20 \mathrm{~ms}$ before the true $O$ point. The systolic upstrokes (c) of the two upper curves are, however, simultaneous. 
are reproduced by the recording system we have chosen to study how sine curves from a loudspeaker are reproduced by the system. The frequencies of these sine curves have been chosen so as to cover the high frequency content that the pulse curves can be assumed to have.

The reproduction in the low frequency range could not be measured by sine curves down to a few tenths of a Hz, since test equipment for this was not available. The reproduction of the slowly changing parts of the curves has, therefore, been studied directly with the low frequency time constant defined below.

\section{HIGH FREQUENCY RANGE}

The amplitude of the recorded curve as a function of frequency is designated $A$, and $A_{0}$ is the amplitude of the curve as a function of frequency recorded without distortion. The degree of distortion is usually expressed as the ratio $A / A_{0}$. The frequency can be normalised by referring it to the resonance frequency $f_{0}$ as a ratio $f / f_{0}$. The ratio $A / A_{0}$ in-

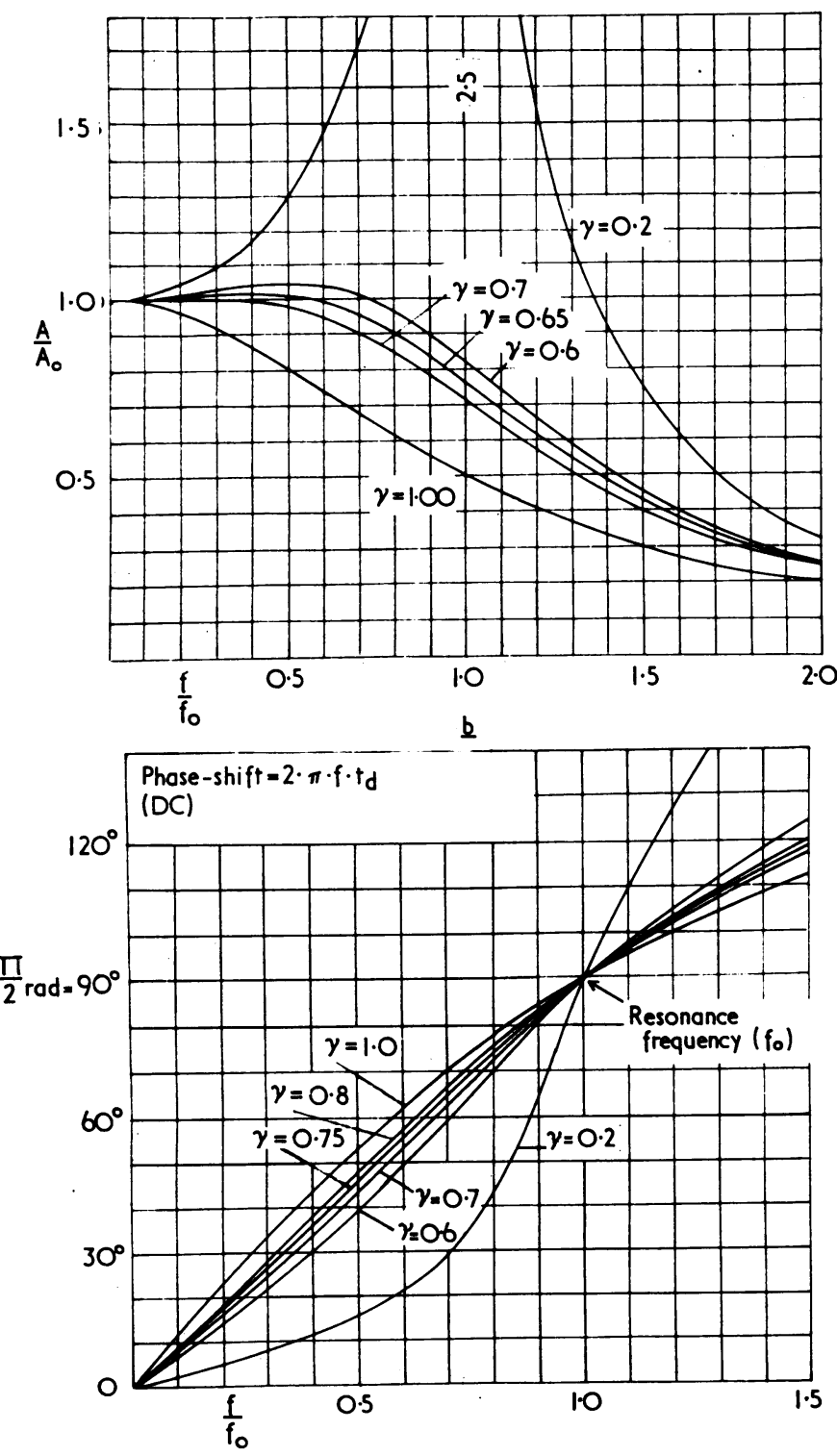

Fig. 2 Frequency characteristics in the high frequency range, typical for the recording systems used in non-invasive cardiology. (a) Theoretical diagram showing the effect of varying degree of damping $\gamma$ on the amplitude/frequency characteristics. (b) Theoretical diagram showing the effect of varying degree of damping on the phase/frequency characteristics. Modified by permission, SPRI, 1970 (abbreviations, see Methods). 
creases towards the resonance frequency, where the overamplification of the recorded curve is at its maximum $A_{\text {max }}$. After the resonance peak the ratio $\mathrm{A} / \mathbf{A}_{\mathbf{0}}$ declines again (Fig. 2 and 3 ). Within the range of measurement $A / A_{0}$ should be as close to 1 as possible. In order to achieve this, damping of the system is usually required, which can be achieved by including a capillary in the air transmission in the system. The degree of damping in the curve, with the system used, can be described by the damping factor $\gamma$ (Fig. 2b and 3). The upper frequency limit $f_{h}$ is defined as the frequency at which $\mathrm{A} / \mathbf{A}_{0}=1 / \sqrt{ } 2(=0.7),-3 \mathrm{~dB}$. A precondition, however, is that overamplification does not give resonance peaks with $\mathrm{A} / \mathrm{A}_{0}>\sqrt{ } 2(=1 \cdot 4)$, $+3 \mathrm{~dB}$.

In addition to the amplitude/frequency characteristics described above the phase/frequency characteristics of the system of recording must be described (Fig. 2b). Phase-shift means that the curve recorded will be shifted in time in relation to the original curve (Fig. 4). Since reference tracings are often used in order to calculate time intervals it is desirable to achieve a constant time shift not varying with frequency content of the recorded curve, which can be achieved by capillary damping. The phase shift is expressed in degrees and can be converted to

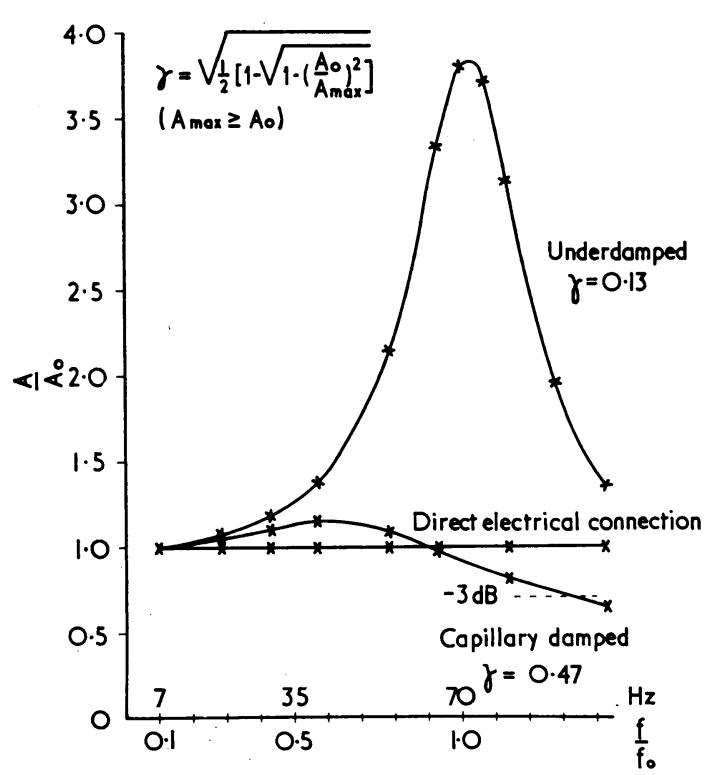

Fig. 3 Amplitude/frequency characteristics for transducer 4 for under-damped, capillary-damped, and direct electrically connected sine curve in the frequency range 7 to $100 \mathrm{~Hz}$. The upper frequency limit (-3dB) for the capillary-damped curve was in this case $95 \mathrm{~Hz}$. The formula for the damping factor $\gamma$ is given in the Fig. time in milliseconds as the phase shift in milliseconds equals (phase shift in degrees $\times 1000$ ): $(\mathrm{f} \times 360)$.

The best phase linearity is achieved with the damping factor $\gamma=0.75$ (Fig. 2b). The best reproduction of the amplitude is achieved with $\gamma=0.65$ (Fig. 2a). As a compromise between phase and amplitude reproduction $\gamma=0.7$ may be used and the phase shift is then approximately a linear function of the frequency, which means a constant delay time. At the resonance frequency the phase shift is the phase shift is $\pi / 2$ radians. The delay time $t_{d}$ can then easily be calculated if the resonance frequency for the system is determined as $t_{d}=1:\left(4 \times f_{0}\right)$ (Fig. $\left.2 b\right)$. In addition to this delay time there is a delay caused by the length of the tube between the pick-up and the transducer, which can be calculated on the basis of the velocity of sound, $340 \mathrm{~m} / \mathrm{s}$.

\section{LOW FREQUENCY RANGE}

The low frequency limit $f_{1}$ with $A / A_{o}=0 \cdot 7,-3 d B$ can be described by means of the low frequency time constant $T C$, the relation being $f_{1}=1:(2 \pi T C)$ (Fig. 5). The low frequency time constant is governed by a filter, usually a so-called RC filter in the transducer (Kastor et al., 1970).

The phase shift in the low frequency range is dependent on the low frequency time constant and differs in character from that in the high frequency range. A short low frequency time constant causes a phase shift of, particularly, the low frequency parts of a curve and thus gives an apparent prematurity of certain points, e.g. the $O$ point of the apex cardiogram ( $O$ stands for mitral valve opening) (Fig. 1). The shorter the low frequency time constant and the slower the curve form, the closer the maxima and minima of the phase-displaced curve will be to the steepest part of the original curve. The maxima and minima in the phasedisplaced curve thus reflect parts of the curve different from the maxima and minima in the original curve. This phase shift, which gives an apparent prematurity, is in this paper called the 'displacement' in order to distinguish it from the phase shift in the high frequency range which gives delay. The phase shift can be calculated for different frequencies if the low frequency time constant or low frequency limit is known (Fig. 5).

\section{Methods}

The dimensions of the capillary-damped pick-up are shown in Fig. 6. With the properties of the underdamped system known, the dimensions of the capillary were chosen on a theoretical basis so as to 


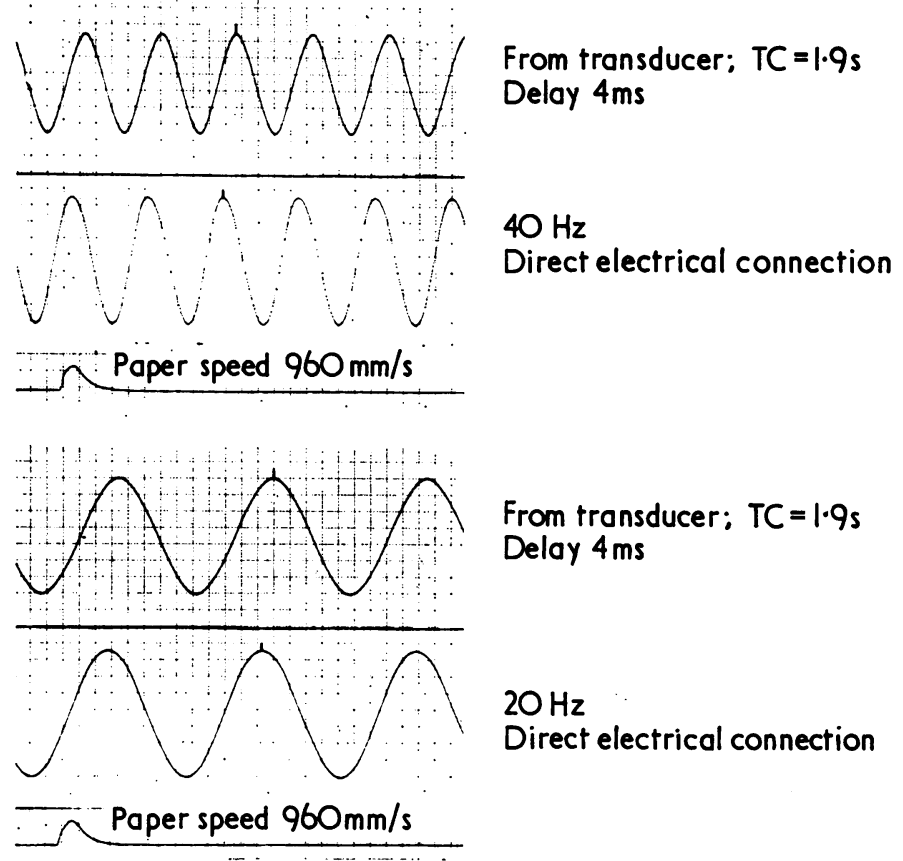

Fig. 4 Sine curves with frequency of 20 and $40 \mathrm{~Hz}$, respectively, recorded by direct electrical connection from the frequency generator to the mingograph and simultaneous recording via transducer No. 1 by placing the capillary-damped funnel over the calibration unit, which was also connected to the frequency generator. Note that the delay is about $4 \mathrm{~ms}$ at both 20 and $40 \mathrm{~Hz}$. The low frequency time constant TC was in this case $1.9 \mathrm{~s}$.

give a damping factor $\gamma$ of about $0 \cdot 7$ (see Appendix). The diameter of the mouth of the funnel was selected to be $25 \mathrm{~mm}$ for practical reasons in order to achieve optimum contact for recording the apex cardiogram and pulse curves such as the carotid pulse, jugular venous pulse, and hepatic pulsation. The funnel was connected via a 35 to $40 \mathrm{~cm}$ long thick-walled rubber tube to the transducer. Too short a tube makes it difficult to achieve the freedom of movement required when recording the curves.

Four complete recording units consisting of a capillary-damped funnel, tube, and transducer were investigated. For purposes of comparison all transducers were also investigated with the pick-up without capillary damping. The two types of pickups were also investigated with a shorter tube length of $20 \mathrm{~cm}$.

The transducer $510 \mathrm{C}$ (Siemens-Elema AB, Sweden) is a crystal transducer. The degree of amplification in this crystal transducer is regulated in steps by a capacitive voltage attenuator so that the low frequency time constant varies with the amplification. The transducer has 11 settings and the highest sensitivity is obtained at setting 11 .

The curves were recorded on a direct-writing mingograph (EM 81, Siemens-Elema AB, Sweden) with linear frequency response between 0 and $500 \mathrm{~Hz}$ and 30 per cent amplitude reduction at $650 \mathrm{~Hz}$. The mingograph was equipped with a differentiation unit (EMT 64).

The amplitude/frequency characteristics were determined by connecting the pick-up to a loudspeaker (type $6 \mathrm{CIS} / \mathrm{B}, 50 \mathrm{ohm}$, Audax, France).

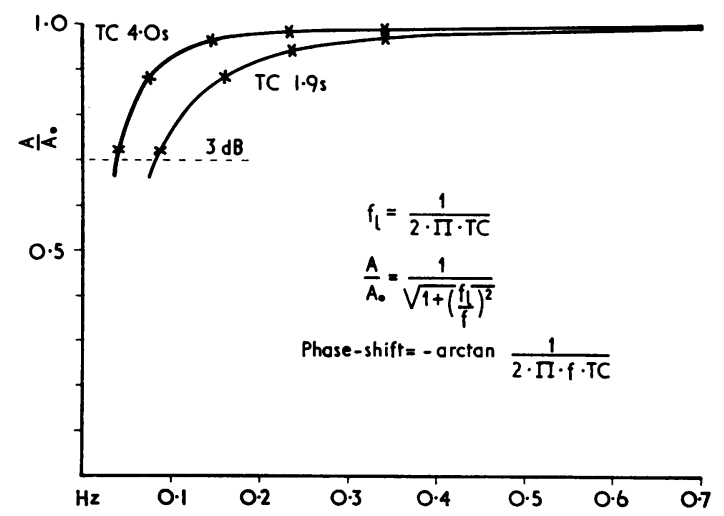

Fig. 5 Theoretical construction of amplitude/frequency characteristics in the frequency range below $0.7 \mathrm{~Hz}$ at low frequency time constants TC 1.9 and $4.0 \mathrm{~s}$. The low frequency limit $f_{l}$ (indicated by dashed line) is determined by the low frequency time constant, as shown by the formula in the figure. The formula which defines the relation between the phase shift, low frequency time constant, and frequency is also given (abbreviations, see 'theoretical background'). 


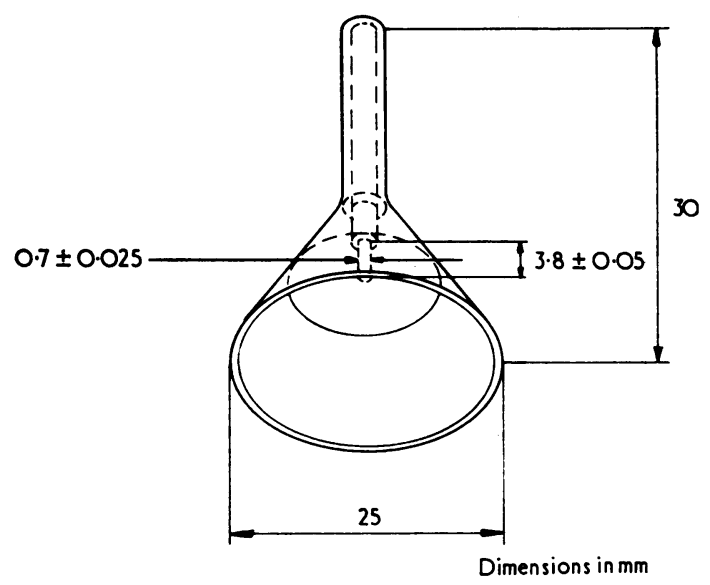

Fig. 6 Design of the capillary-damped pick-up.

The loudspeaker was sealed by painting it twice (Oppanol B-dispersion, approximately $60 \%$, BASF, Western Germany) and intended for calibration of non-invasive curves (Fig. 7). It normally gave a triangular wave with a frequency of $10 \mathrm{~Hz}$ (Fig. 8). In the study, however, the loudspeaker was connected to a frequency generator (Wavetek Function Generator, Model 110, San Diego, U.S.A.) giving sine impulses with increasing frequency at intervals of $1 \mathrm{~Hz}$ below $10 \mathrm{~Hz}$ and at intervals of $5 \mathrm{~Hz}$ above $10 \mathrm{~Hz}$. The frequency generator was also electrically connected direct to the mingograph.

If the loudspeaker receives a constant current a constant force acts on the cone, and the cone, which is spring-loaded, moves a constant distance, i.e. gives a constant volume as long as the frequency is well below the resonance frequency of the loud-

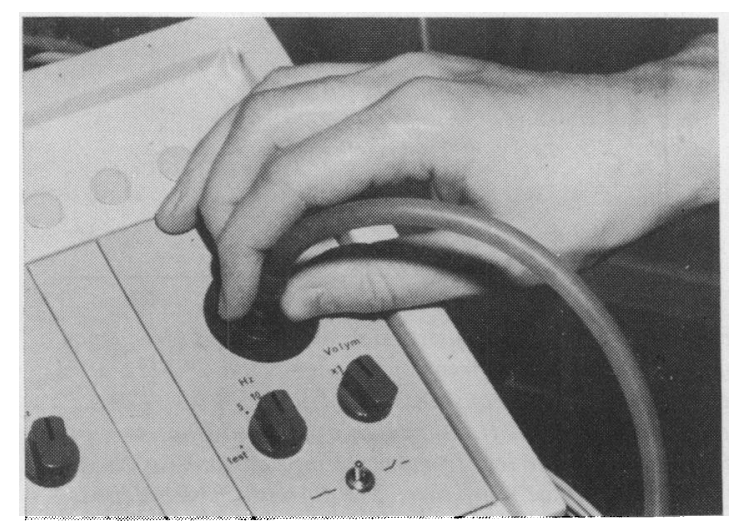

Fig. 7 The capillary-damped funnel with its transducer coupled to the calibration unit for calibration. speaker. Thus, once a pick-up had been placed over the loudspeaker, the loudspeaker gave constant volume changes in the frequency range 7 to $100 \mathrm{~Hz}$, as the resonance frequency of the loudspeaker was above $200 \mathrm{~Hz}$. In order to avoid leakage of air, which would distort the curve by shortening the low frequency time constant (Kastor et al., 1970), the funnel was placed against a rubber strip on the loudspeaker (Fig. 7). Below the frequency 5 to $7 \mathrm{~Hz}$ a reduction of amplitude was observed, probably owing to the fact that the loudspeaker could not be completely sealed.

From the experimentally-obtained amplitude/ frequency response the degree of damping $\gamma$ was calculated (Fig. 3). The delay time $t_{d}$ owing to phase shift was calculated on the basis of the resonance frequency from the expression $t_{d}=$ $1:\left(4 \times f_{0}\right)$, Fig. $2 b$.

The low frequency time constant was determined as the time it took for the curve to revert to 37 per cent $(1 / e)$ of the total deflection obtained in connection with the sudden release of a positive pressure exerted on the transducer. This method proved more practical than sudden application of a positive pressure to the transducer, which is the normal procedure. On the basis of the low frequency time constant the low frequency limit $f_{1}$ was determined (Fig. 5).

\section{Results}

The resonance frequency in the underdamped system varied between 70 and $90 \mathrm{~Hz}$ (Table 1). The maximum amplitude distortion varied in the underdamped system between $2 \cdot 4$ and $4 \cdot 3$. These figures thus describe the overamplification. In the capillary-damped system the overamplification de-

Table 1 Resonance frequency $\left(f_{o}\right)$ for underdamped system, high-frequency limit $\left(f_{\mathrm{h}}\right)$ for capillary-damped system, maximal amplitude distortion $\left(A_{\max } / A_{0}\right)$ for capillary-damped and underdamped system, and corresponding calculated damping factor $\gamma$ for the four recording units

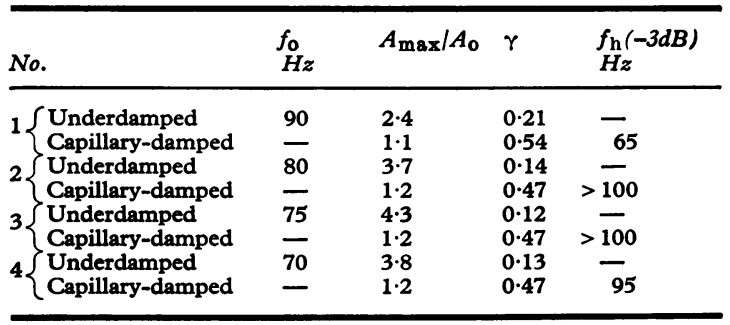

The parameters $A_{0}, A_{\max }, f_{0}$, and $f_{h}$ were measured. The damping factor $\gamma$ was calculated from the formula given in Fig. 3. 

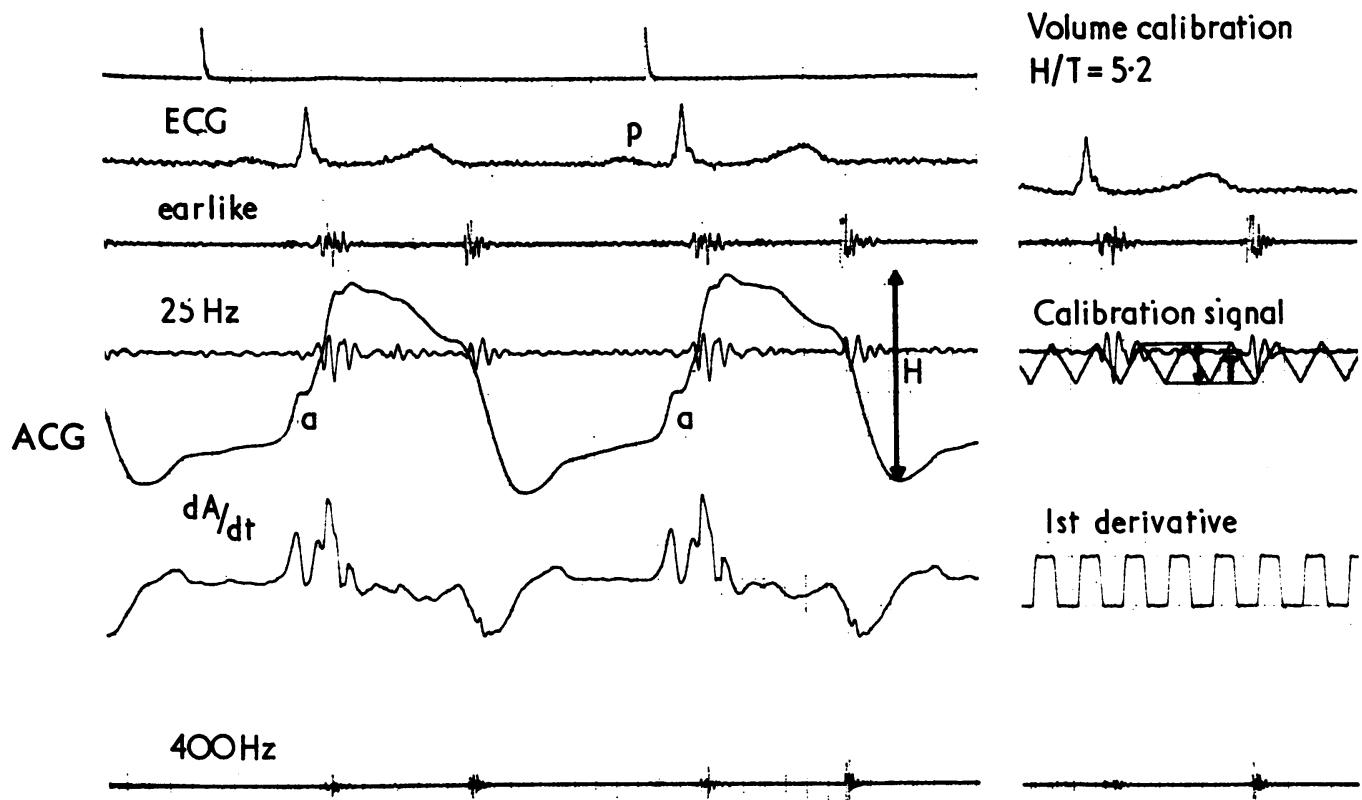

Fig. 8 Calibration of the apex cardiogram recorded simultaneously with electrocardiogram (lead II) and phonocardiogram (ear-like, 25 and $400 \mathrm{~Hz}$ ) (right-hand panel). The total height $(\mathrm{H})$ of the apex cardiogram, the amplitude of the a wave (a) (left-hand panel), and the amplitude of the calibration signal $(T)$ are marked. The first derivative of the apex cardiogram (dA/dt) and of the calibration signal was also recorded. The calibration gave $H / T=5 \cdot 2$ arbitrary units.

creased to $1 \cdot 1$ to $1 \cdot 2$. The high frequency limit was above $100 \mathrm{~Hz}$ in two of the systems, and 65 and $95 \mathrm{~Hz}$, respectively, in the other two. The damping factor in the underdamped system varied between $0 \cdot 12$ and 0.21 , while in the capillary-damped system it was improved to 0.47 to 0.54 . The amplitude/ frequency characteristics for transducer number 4 for the underdamped and capillary-damped curve in the frequency range 7 to $100 \mathrm{~Hz}$ are shown in Fig. 3.

If the length of the tubing was reduced to $20 \mathrm{~cm}$ the resonance frequency in the underdamped system increased by about $10 \mathrm{~Hz}$. No significant differences were obtained in this respect in the capillary-damped system.

At high frequencies and assuming that capillary damping produced an approximately linear function between frequency and phase shift, the delay time $t_{d}$ was calculated on the basis of the resonance frequency to be 2.8 to $3.6 \mathrm{~ms}$. With tube lengths between 35 and $40 \mathrm{~cm}$ there was an additional delay of 1.0 to $1.2 \mathrm{~ms}$. The total delay in the system described was thus on theoretical grounds on average $4 \mathrm{~ms}$, and the experiments in Fig. 4 show also that the delay time was about $4 \mathrm{~ms}$ and essentially the same at 20 and $40 \mathrm{~Hz}$.
The low frequency time constant was determined for the four transducers at settings 3 to 11 (Table 2). $f_{l}$ varied from $0.035 \mathrm{~Hz}$ at low frequency time constant $4.6 \mathrm{~s}$ to $0.094 \mathrm{~Hz}$ at time constant $1.7 \mathrm{~s}$.

Table 3 shows the theoretically calculated displacement of sine curves at frequencies between 0.5 and $2 \mathrm{~Hz}$ with low frequency time constants of 1.9 and $4.6 \mathrm{~s}$. Because of the displacement the maxima and minima of the phase-shifted sine curve will occur 53 to $1 \mathrm{~ms}$ before the maxima and minima of the original curve, depending on

Table 2 Low frequency time constants for four transducers at amplifier settings from 3 to 11

\begin{tabular}{|c|c|c|c|c|}
\hline Setting & $\begin{array}{l}\text { Transducer nu } \\
1 \\
\text { Low frequenc }\end{array}$ & $\begin{array}{l}\text { number } \\
2 \\
\text { cy time }\end{array}$ & $\begin{array}{l}3 \\
\text { stant }(s)\end{array}$ & 4 \\
\hline $\begin{array}{r}3 \\
4 \\
5 \\
6 \\
7 \\
8 \\
9 \\
10 \\
11\end{array}$ & $\begin{array}{l}2.0 \\
2.0 \\
2.1 \\
2.2 \\
2.4 \\
2.6 \\
2.9 \\
3.7 \\
1.9\end{array}$ & $\begin{array}{l}2 \cdot 1 \\
2 \cdot 1 \\
2 \cdot 2 \\
2 \cdot 3 \\
2 \cdot 4 \\
2 \cdot 8 \\
3 \cdot 1 \\
4 \cdot 6 \\
2 \cdot 3\end{array}$ & $\begin{array}{l}1.7 \\
1.7 \\
1.7 \\
1.8 \\
2.0 \\
2.3 \\
2.6 \\
3.8 \\
2.1\end{array}$ & $\begin{array}{l}2 \cdot 0 \\
2 \cdot 0 \\
2 \cdot 1 \\
2 \cdot 1 \\
2 \cdot 4 \\
2 \cdot 6 \\
3 \cdot 0 \\
4 \cdot 0 \\
2 \cdot 2\end{array}$ \\
\hline
\end{tabular}


Table 3 Theoretically calculated displacement of sine curve at frequencies from 0.5 to $2.0 \mathrm{~Hz}$ at low frequency time constant TC 1.9 and $4.6 \mathrm{~s}$

\begin{tabular}{lll}
\hline $\begin{array}{l}\text { Frequency } \\
\mathrm{Hz}\end{array}$ & TC 1.9s & TC 4.6s \\
& Displacement $\mathrm{ms}$ & \\
\hline 0.5 & -53 & -22 \\
1.0 & -13 & -6 \\
1.5 & -6 & -2 \\
2.0 & -3 & -1 \\
\hline
\end{tabular}

The phase shift was calculated from the formula presented in Fig 5 (conversion from phase shift in degrees to displacement in $\mathrm{ms}$, see text).

frequency and the low frequency time constant. Note that the displacement increases greatly at short low frequency time constants and slow frequencies.

The theoretical construction of amplitude/ frequency characteristics in the frequency range below $0.7 \mathrm{~Hz}$ for the low frequency time constants TC 1.9 and $4 \mathrm{~s}$ are shown in Fig. 5. The amplitude/ frequency response was assumed to be flat in the range 0.7 to $7 \mathrm{~Hz}$. The conclusion that a significant leakage was not present above $7 \mathrm{~Hz}$ was based upon the finding that the amplitude of the recorded curve was almost constant between 5 and $10 \mathrm{~Hz}$ (see Methods).

Fig. 1 shows an apex cardiogram recorded with low frequency time constant $2.3 \mathrm{~s}$ and with curve processing giving a low frequency time constant of $0.3 \mathrm{~s}$, and the first derivative of the apex cardiogram. The electrocardiogram and phonocardiogram are recorded simultaneously. With the too short low frequency time constant $0.3 \mathrm{~s}$ (fifth channel), pronounced sag is displayed in the plateau of the apex cardiogram. This sag causes an undershoot of the following baseline which leads to a premature, false $O$ point. In the apex cardiogram recorded with low frequency time constant $0.3 \mathrm{~s}$ the true $O$ point is in fact impossible to identify, and measurement of the interval between the aortic component of the second heart sound $A_{2}$ and the $O$ point in the apex cardiogram gave in this example an underestimate of the $\mathrm{A}_{2} \mathrm{O}$ interval by about $20 \mathrm{~ms}$. The systolic upstroke (c) of the first derivative was delayed by about $4 \mathrm{~ms}$ in relation to the analogous point in the apex cardiogram, depending on the filter used for differentiation. In the two upper curves the systolic upstrokes $c$ are simultaneous but the $O$ points of the two curves are not. This is because of the low frequency time constant and the different frequency content in those parts of the apex cardiogram which contain the systolic upstroke $c$ and $O$ point respectively.

\section{Discussion}

The results show that the capillary-damped funnel gave a considerable reduction of the amplitude distortion and a constant phase shift in the high frequency range though the damping achieved was not optimal.

For invasively recorded curves using a micromanometer in the left ventricle a high frequency limit between 40 and $80 \mathrm{~Hz}(-3 B)$ depending on heart rate and left ventricular function is desirable for correct reproduction (Broman et al., 1975). The high frequency content in the non-invasive curves is probably smaller than in those recorded invasively, however. For recording of the apex cardiogram a high frequency limit of $50 \mathrm{~Hz}$ has been suggested since the high frequency content according to Fourier analysis at high heart rates may reach this frequency (Kesteloot et al., 1969; Denef et al., 1975). In the capillary-damped system the high frequency limit was never lower than $65 \mathrm{~Hz}$, which is probably quite sufficient for the purposes.

The amplitude of the curve recorded without capillary damping was distorted, especially in the high frequency range. Theoretically this distortion seems to be of importance. This is illustrated by a comparison between the amplitude/frequency responses for the underdamped and capillary-damped system in relation to an assumed calibration signal. The high frequency content of a recorded curve will practically always be unknown and will vary from individual to individual according to heart rate and left ventricular function. This means that the results of the calibration, especially for the first derivative, will also vary with the high frequency content in the curve: owing to the variation in amplitude distortion the calibration figure will be very different, for example, at 10 and $50 \mathrm{~Hz}$, since the frequency of the calibration signal is constant, for example $10 \mathrm{~Hz}$. This has not, however, been studied experimentally in this paper.

Further improvement of the amplitude/frequency characteristics is desirable. This has been achieved with a sophisticated combined microphone for recording pulse curves, electrocardiogram, and phonocardiogram described in another paper (Nilsson and Thulesius, 1973). This has, however, been achieved at the cost of a somewhat heavier and larger pick-up, which makes recording of the apex cardiogram more difficult than with the funnelformed pick-up in cases in which palpation of the heart beat is difficult. Construction of a smaller funnel-shaped pick-up with the same good physical properties as the combined microphone is in progress.

The calculated delay at high frequencies was 
based on the assumption that there was an approximately linear relation between frequency and phase shift. There is a mathematical relation between the phase shift, the damping factor, and the resonance frequency of the recording system used (Doebelin, 1966). An idea of the limits within which phase shift varied in the underdamped curve can be obtained from the fact that at a damping factor of $\gamma=0 \cdot 13$, corresponding to $A_{\max } / A_{0}=3.8$ and a resonance frequency of $70 \mathrm{~Hz}$ (system 4), the delay varies between 0.6 and $3.3 \mathrm{~ms}$, for the frequencies 5 and $70 \mathrm{~Hz}$, respectively.

The total delay caused by phase shift in the capillary-damped system was on average $4 \mathrm{~ms}$ at high frequencies. This delay gives a prolongation or shortening of a studied time interval depending on whether the measurement point on the reference signal occurs before or after the measurement point on the phase-shifted curve. When calculating the total shift, consideration must be given to both this delay and to the displacement resulting from the low frequency time constant.

In the apex cardiogram the $\mathrm{a} / \mathrm{H}$ ratio can be accurately calculated even if the low frequency time constant is as short as $0.06 \mathrm{~s}$ despite the form of the curve being seriously distorted (Johnson et al., 1971). In order to be able to calculate the interval between the aortic component of the second heart sound and the O-point in the apex cardiogram with negligible distortion, on the other hand, the low frequency time constant should be in the vicinity of $2 \mathrm{~s}$. If it is assumed that no measurement segments of the non-invasive curves have a lower frequency content than $2 \mathrm{~Hz}$ (Kesteloot et al., 1969), the displacement caused by the low frequency time constant will for a low frequency time constant $2 \mathrm{~s}$ be less than $3 \mathrm{~ms}$ and for a low frequency time constant above $4.6 \mathrm{~s}$ it will be less than $1 \mathrm{~ms}$ (Table 3).

Transducer settings below 7 are in practice very seldom used when the transducer is connected to the DC input in the mingograph and then the low frequency time constant was not below $1.9 \mathrm{~s}$, giving a low frequency limit of $0.084 \mathrm{~Hz}$. If a transducer is connected to an AC input in an electrocardiogram amplifier, which is not uncommon during recording of pulse curves, this may cause shortening of the low frequency time constant (Kastor et al., 1970). This cannot be neglected, since the low frequency time constant in electrocardiograph amplifiers is often in the vicinity of $1 \mathrm{~s}$.

Kesteloot et al. (1969) introduced the concept 'dominant frequency' and used this for analysis of phase distortion in the low frequency range. Dominant frequency has been defined as the frequency of a sine wave which would have the same phase shift as that part of the curve under study. As pointed out under 'theoretical background', the maxima and minima in the phase-shifted curve reflect different parts of the curve from the maxima and minima in the original curve. This difference is accentuated the shorter the low frequency time constant and the slower the curve form is. With pronounced distortion of the apex curve the point of intersection of the most rapid segment of the declining part of the apex cardiogram will influence the position of the false $O$ point (Fig. 1). If the low frequency time constant is too short the recorded $\mathrm{A}_{2} \mathrm{O}$ interval will depend on the point of time at which this most rapid part of the curve occurs quite independently of the dominant frequency for the area around the true O-point. This phenomenon might influence the outcome of dominant frequency analyses.

Measurement of the interval between the second heart sound and the $O$ point in the apex cardiogram with too short a low frequency time constant is thus open to serious error. Data from our group showed a prolongation of this interval to above $150 \mathrm{~ms}$ in more than 50 per cent of patients who had essential hypertension and in above 70 per cent of patients who had sustained a myocardial infarct (Wikstrand et al., 1977). One reason why such a striking finding has been so little studied may be the use of transducers with too short low frequency time constants in many previous studies, since transducers with short low frequency time constant not only shorten the $\mathrm{A}_{2} \mathrm{O}$ interval but tend to smooth out differences between an individual with a prolonged and a person with a normal $\mathrm{A}_{2} \mathrm{O}$ interval (Johnson et al., 1971). Changing the low frequency time constant, for example, from $2.5 \mathrm{~s}$ to $0.06 \mathrm{~s}$ in that paper gave a reduction of the mean difference of $30 \mathrm{~ms}$ between two groups to $15 \mathrm{~ms}$, which in this connection is a substantial change (Wikstrand et al., 1977).

The frequency content in a segment of a curve is probably dependent on both heart rate and left ventricular function and probably increases with increasing heart rate and decreases with impaired left ventricular function. Studies of the frequency content for the curves should be carried out for both low frequency and high frequency segments. On the basis of this type of study, norms can be established for characteristics of transducers to be used for recording of non-invasive cardiac pulse curves. Standardisation of these norms is desirable. Data from different scientific centres are at present sometimes difficult to compare owing to great variations and often unknown physical properties in the systems used for recording the pulse curves.

In addition to calculation of time intervals and 
$\mathrm{a} / \mathrm{H}$ ratios, further quantitative non-invasive data concerning cardiac function can probably be obtained by calibration and differentation of the curves. As regards the apex cardiogram, calibration and differentiation of both systolic and diastolic curve segments may be of value (Denef et al., 1975; Denef and Kesteloot, 1976). Good amplitude/ frequency characteristics and phase/frequency characteristics in the system of recording constitute one of the preconditions for successful results with such studies.

\section{Appendix}

\section{Approximate calculation of damping capillary dimensions}

The damping capillary forms together with the cavity an RC network. The output of this network is connected to the transducer. Fig. 9 shows a simplified sketch of the system.

\section{CALCULATION OF RC NETWORK CUTOFF} FREQUENCY ( $-3 \mathrm{~dB}$ limit)

(Ia) Damping capillary flow resistance

We assume that a laminar flow always prevails in the capillary owing to the low flow speed in the capillary and make use of Poiseuille's expression for laminar flow in a capillary:

$$
\frac{\Delta \mathrm{P}}{\mathrm{Q}}=\frac{8 \eta 1}{\pi \mathrm{r}^{4}}
$$

where $\mathrm{Q}$ indicates volume per unit time, $\Delta \mathrm{P}$ the fall in pressure across the capillary, $r$ the radius of the capillary, $\eta$ the gas viscosity, and 1 the length of the capillary.

The electrical equivalent regards $\Delta \mathrm{P}$ as a voltage drop and $Q$ as a current. The expression $\Delta P / Q$ can therefore be defined as the flow resistance $R$ :

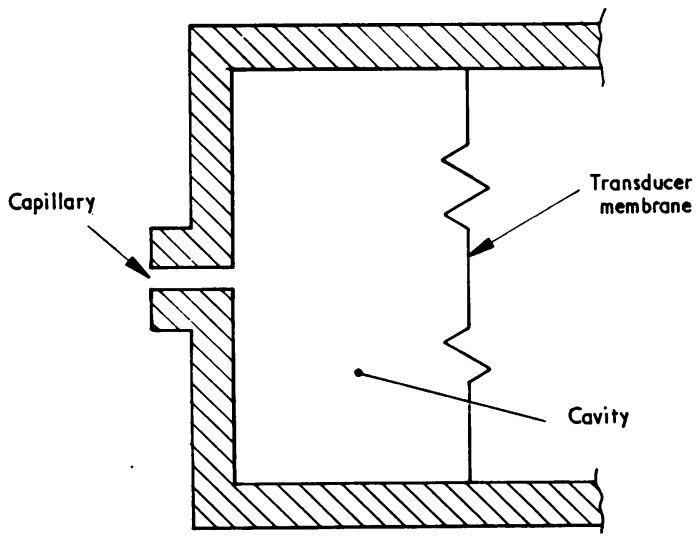

Fig. 9 Simplified sketch of the capillary-damped system.

$$
\mathrm{R}=\frac{8 \eta 1}{\pi \mathrm{r}^{4}}
$$

\section{(Ib) Cavity compliance}

As we are dealing with rapid pressure changes in the cavity, negligible heat exchange will occur and we make use of the expression for an adiabatic process:

$$
\mathrm{PV}^{\mathrm{K}}=\text { constant }
$$

where $P$ is the pressure and $V$ the sum of tube volume and internal transducer volume. $K=1 \cdot 4$ for air.

Differentiation results in

$$
\begin{gathered}
P K V^{k-1} d V+V^{k} d P=0 \\
\frac{d V}{d P}=-\frac{1}{K} \cdot \frac{V}{P}
\end{gathered}
$$

$\mathrm{dV} / \mathrm{dP}$ is the cavity compliance $\mathrm{C}$, whose electrical equivalent is a capacitance.

(Ic) Cutoff frequency

The $R C$ network cutoff frequency $f_{c}$ is now given by

$$
\mathrm{f}_{\mathrm{c}}=\frac{1}{2 \pi \mathrm{RC}}=\frac{\mathrm{KPr}^{4}}{16 \eta \mathrm{V} 1}
$$

With given values of $f_{c}$ and $V$, a suitable capillary can be calculated. $K, \eta$ are constants, $P$ the barometer pressure.

\section{TOTAL FREQUENCY RESPONSE}

The compliance of the transducer membrane is assumed to be much smaller than the cavity compliance, so that the transducer loading of the RC network output is neglected.

The total frequency response is then given by the sum of the network response (in $\mathrm{dB}$ ) and the transducer response (in $\mathrm{dB}$ ).

This study was supported by grants from the Swedish Medical Research Council (K73-19xx4131-01, B74-19x-4229-01A), from the Swedish National Association against Heart and Chest Diseases, and the Bank of Sweden Tercentenary Fund.

The critical help given by Professor $\mathrm{H}$. Wallman, Medical Electronics, Chalmer's University of Technology, and the technical assistance given by Tord Bergerheim is appreciated.

\section{References}

Broman, H., Kvasnicka, J., Liander, B., and Varnauskas, E. (1975). A computerized system for optimal filtering of left ventricular pressure data. IEEE Transactions on Biomedical Engineering, BME-22, 4, 287-292.

Denef, B., and Kesteloot, H. (1976). Study of diastolic heart sounds by means of calibrated apexcardiography (abstract). European fournal of Cardiology, 4, 398. 
Denef, B., Popeye, R., de Geest, H., and Kesteloot, H. (1975). On the clinical value of calibrated displacement apexcardiography. Circulation, 51, 541-551.

Doebelin, E. (1966). Measurement Systems: Application and Design, p. 134. McGraw-Hill, New York.

Johnson, J. M., Siegel, W., and Blomqvist, G. (1971). Characteristics of transducers used for recording the apexcardiogram. Fournal of Applied Physiology, 31, 796800.

Kastor, J. A., Aronow, S., Nagle, R. E., Garber, T., and Walker, H. (1970). Air leaks as a source of distortion in apexcardiography. Chest, 57, 163-169.

Kesteloot, H., Willems, J., and van Vollenhoven, E. (1969). On the physical principles and methodology of mechanocardiography. Acta Cardiologica, 24, 147-160.

Nilsson, K., and Thulesius, O. (1973). A combined microphone for simultaneous recording of pulse and heart sounds. Bio-Medical Engineering, 8, 424-431.

Reale, A. (1967). Evaluation of the contractile state of the human heart from the first derivative of the apexcardiogram. Circulation, 36, 933-941.
SPRI Advice 6.5 (December 1970). Blood pressure transducer-testing. SPRI, Stockholm.

Sutton, G. C., and Craige, E. (1967). Quantitation of precordial movement. I. Normal subjects. Circulation, 35, 476-482.

Sutton, G. C., Prewitt, T. A., and Craige, E. (1970). Relationship between quantitated precordial movement and left ventricular function. Circulation, 41, 179-190.

Wikstrand, J., Wilhelmsen, L., Berglund, G., and Wallentin, I. (1977). The value of systolic and diastolic time intervals. Studies in normotensive and hypertensive 50-year-old men and in patients after myocardial infarction. British Heart fournal. (In press.)

Requests for reprints to Dr. John Wikstrand, Department of Clinical Physiology, Sahlgren's Hospital, University of Göteborg, S-413 45 Göteborg, Sweden. 